\title{
Universal aspects of hydrogel gelation kinetics, percolation and viscoelasticity from PA-hydrogel rheology
}

Vahid Adibnia and Reghan J. Hill

Citation: Journal of Rheology 60, 541 (2016); doi: 10.1122/1.4948428

View online: http://dx.doi.org/10.1122/1.4948428

View Table of Contents: http://sor.scitation.org/toc/jor/60/4

Published by the The Society of Rheology

\section{Articles you may be interested in}

Thermoreversible gelation and viscoelasticity of $\kappa$-carrageenan hydrogels Journal of Rheology 60, (2016); 10.1122/1.4938525

i-Rheo: Measuring the materials' linear viscoelastic properties "in a step"! Journal of Rheology 60, (2016); 10.1122/1.4953443

Start-up shear of concentrated colloidal hard spheres: Stresses, dynamics, and structure Journal of Rheology 60, (2016); 10.1122/1.4949340

Modeling the effects of polydispersity on the viscosity of noncolloidal hard sphere suspensions Journal of Rheology 60, (2016); 10.1122/1.4938048

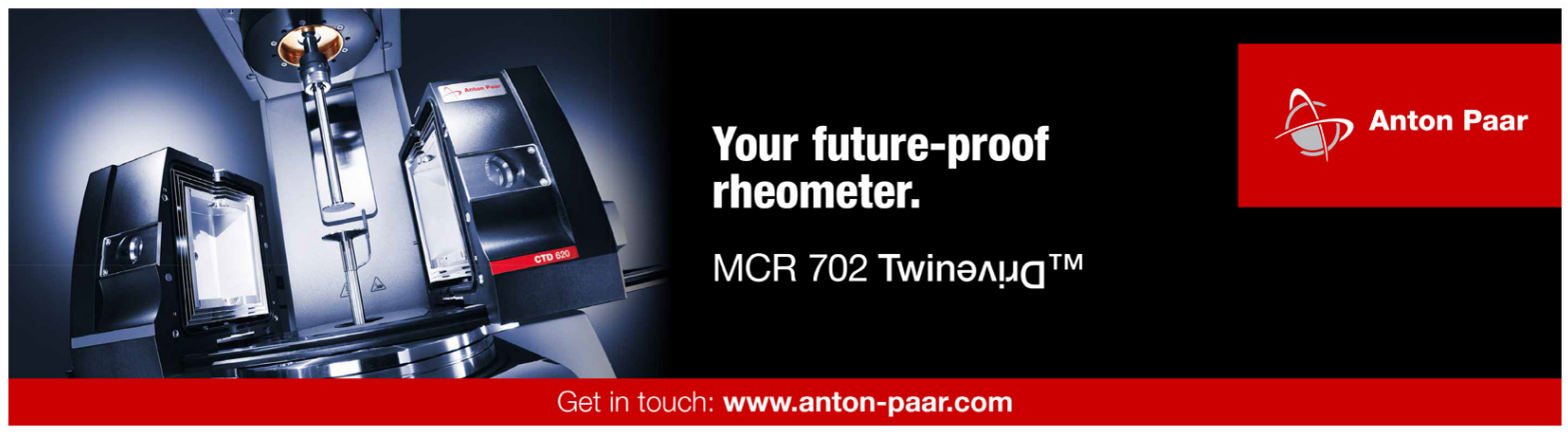




\title{
Universal aspects of hydrogel gelation kinetics, percolation and viscoelasticity from PA-hydrogel rheology
}

\author{
Vahid Adibnia and Reghan J. Hill ${ }^{a)}$ \\ Chemical Engineering, McGill, 3610 University Street, Montreal, Quebec H3A 0C5, Canada
}

(Received 10 February 2016; final revision received 15 April 2016; published 6 May 2016)

\begin{abstract}
Polyacrylamide (PA) hydrogels have been studied extensively, but fundamental aspects of their gelation kinetics, percolation dynamics, and viscoelasticity are still not well understood. This paper focuses on the rheology of PA hydrogels having unusually low monomer concentrations $\left(c_{a} \approx 3 \mathrm{w} \%\right.$ equivalent to $\left.0.42 \mathrm{~mol} 1^{-1}\right)$. These furnish loss tangents that span 4 orders of magnitude when varying the crosslinker concentration. An optimum crosslinker concentration $\left(c_{\text {bis }} / c_{a} \approx 2.5\right.$ mol. \% equivalent to $\left.5.3 \mathrm{w} \%\right)$ is identified, below which the storage modulus $G^{\prime}$ increases almost linearly, and the loss modulus $G^{\prime \prime}$ acquires a local maximum. Above the optimum crosslinker concentration, $G^{\prime}$ and $G^{\prime \prime}$ both plateau, accompanied by a notable decrease in the maximum strain (increase in brittleness) before breaking. The dynamic shear moduli reveal universal dynamics at the gel point, as indicated by (i) scaling exponents $(y=3.1 \pm 0.1, z=2.1 \pm 0.1$ and $\Delta=0.70 \pm 0.02)$ that are consistent with the de Gennes ["On a relation between percolation theory and the elasticity of gels," J. Phys. Lett. 37, L1-L2 (1976)] electrical network analogy, and (ii) a critical relaxation exponent that is close to the Rouse limit $\Delta=2 / 3$ from the scaling theory of Martin. A close correspondence of the exponents with that of Adam and Delsanti [Macromolecules 18, 2285-2290 (1985)] for the radical copolymerization of a different material supports the long-standing hypothesis that dynamics at the gel point are universal for a prescribed gelation mechanism. (C) 2016 The Society of Rheology. [http://dx.doi.org/10.1122/1.4948428]
\end{abstract}

\section{INTRODUCTION}

Polyacrylamide (PA) hydrogels, synthesized by freeradical polymerization of acrylamide with bisacrylamide (bis) as the crosslinker, have interesting and technologically useful properties. Consequently, it is one of the most widely studied water soluble polymers [1-5], and much has been learned from studies of its gelation [6-8], sol-gel transition [9], degree of crosslinking [10], and large-strain deformation [11]. See Denisin and Pruitt [12] for a recent example of how varying the degree of crosslinking in PA affects its stiffness. While many rheological studies have customarily focussed on the steady-state storage modulus $G^{\prime}$, relatively little attention has been given to the loss modulus $G^{\prime \prime}$, gelation kinetics, and sol-gel transition. The present rheological study identifies $G^{\prime \prime}$ as being particularly sensitive to the microstructure, since it reflects the spatial arrangement, interaction, and dynamics of partial chains and clusters.

The gelation mechanism has been studied extensively $[10,13,14]$ and remains an active research area [6,9]. Polymerization begins with the reaction between radicals and monomers, forming growing centers to which monomers diffuse, thus forming clusters that are linked by PA chains to form a connected network (percolating cluster). These clusters are nondraining and richer in bis-because of the propensity of bis to react with itself [15] - than the surrounding "dilute phase," which is depleted of bis and contains elastically ineffective strands, such as dangling chains.

\footnotetext{
a) Author to whom correspondence should be addressed; electronic mail: reghan.hill@mcgill.ca

The equilibrium storage modulus for hydrogels is generally interpreted using the Kuhn theory of rubber elasticity. Under affine deformation,

$$
G^{\prime}=\nu k_{B} T
$$

where $\nu$ is the network strand number density and $k_{B} T$ is the thermal energy. However, fluctuations can lead to nonaffine deformation, which, according to the phantom-network model [16], furnishes

$$
G^{\prime}=(1-2 / f) \nu k_{B} T
$$

where $f$ is the crosslinker functionality and $1-2 / f$ is the fraction of strands that support the stress [16]. For an ideal network (without dangling chains and loops), the crosslink number density is $n=2 \nu / f$, so a network with tetrafunctional connectivity has $[16,17]$

$$
G^{\prime}=n k_{B} T
$$

i.e., the crosslink density $n$ replaces the network strand density $\nu$ in the Kuhn theory.

A sol-gel transition (at the so-called gel point) is characterized by the storage and loss moduli having a single power-law scaling with respect to frequency

$$
G^{\prime} \sim G^{\prime \prime} \sim \omega^{\Delta}
$$

where $\Delta$ has been termed the critical relaxation exponent, relaxation exponent, or viscoelastic exponent $[9,18,19]$. Near the sol-gel transition, many viscoelastic quantities diverge as powers of the distance of the bond probability $p$ from the gel 
point $\epsilon=\left|p-p_{c}\right| / p_{c}$, where $p_{c}$ is the critical bond probability. Below the gel point, the zero shear viscosity scales as

$$
\eta_{0} \sim \epsilon^{-k}
$$

whereas above the gel point the equilibrium shear modulus scales as

$$
G \sim \epsilon^{-z} .
$$

The transition from a liquid or solid to a viscoelastic material, i.e., crossing the gel point from either below or above, is accompanied by a divergence of the longest relaxation time

$$
\tau_{l} \sim \epsilon^{-y}
$$

Using scaling analysis from percolation theory, Martin et al. [20] showed that the exponents are related by

$$
y=z+k \text { and } \Delta=z / y .
$$

They also suggested that the practical limits for $k$ and $\Delta$ are $0 \leq k \leq 1.35$ and $2 / 3 \leq \Delta \leq 1$, with $k=1.35$ and $\Delta=2 / 3$ in the limit of complete hydrodynamic screening (Rouse dynamics), and $k=0$ and $\Delta=1$ in the limit of strong hydrodynamic interaction (Zimm dynamics). Note that the de Gennes [21] analogy between gelation and electrical networks furnishes $z=1.94$ (Martin's $z=2.67$ ), $k=0.75$, and $\Delta=0.72$ [22].

While experiments often furnish scaling exponents that are in the theoretical range, the values can vary with the material and crosslinking mechanism, so the universality of the scaling exponents has been controversial, and is still an area of recent research [9]. For example, Adam and Delsanti [22] found $z=2.1 \pm 0.3, k=0.78 \pm 0.05$, and $\Delta \approx 0.73$ for radical copolymerization (polystyrene/divinylbenzene/benzene), and $z=3.2 \pm 0.6, k=0.78 \pm 0.05$, and $\Delta \approx 0.80$ for polycondensation (diisocyanate/triol). Adolf and Martin [23] obtained $z=2.8 \pm 0.2, y=3.9 \pm 0.2, \quad$ and $\quad \Delta=0.72 \pm 0.09 \quad(k=1.1$ \pm 0.4 ) from time-cure superposition during crosslinking of epoxy resins. Many other experimental values were reviewed by Martin et al. [19], often furnishing $0.7 \leq k \leq 1.4$ and $0.6 \leq \Delta \leq 0.8$. However, several studies with excess crosslinker at the gel point furnish $\Delta \approx 0.5$. In these cases, comparison to the percolation theory may not be justified because of structural complexity arising from excess crosslinker [19].

Determining of the sol-gel transition from $G^{\prime}$ and $G^{\prime \prime}$ spectra is well described in the literature $[18,19]$ and provides a valid characterization of the gel point. However, bulk rheology has limitations for weak networks and when the gelation kinetics are rapid. To characterize the gelation of these materials, Larsen and Furst [9] used multiple-particletracking microrheology and studied the time-cure superposition (of peptide and PA hydrogels). They constructed a master curve for the mean-squared displacements (MSD) of tracer particles and identified a gel point from the shift factors. For a PA hydrogel, they reported $z=2.3 \pm 0.1$ and $y=$
$3.6 \pm 0.1$ (furnishing $\Delta \approx 0.64$ ) for tracers in the pregels, and $z=1.7 \pm 0.1$ and $y=3.7 \pm 0.2(\Delta \approx 0.46)$ for the postgels.

While the exponents of Larsen and Furst [9] for their pregels are in reasonable agreement with Adam and Delsanti [22] for radical copolymerization, the critical exponent (either $\Delta=0.46$ for the postgel or 0.55 for the averaged preand postgel values) is not in the expected theoretical range $\Delta=2 / 3-1$, and the pre- and postgel values are clearly different. These discrepancies are an important motivation for the present study, which addresses the sol-gel transition in PA hydrogels using a rheometer that is capable of resolving changes in both shear moduli when increasing the pregel crosslinker concentration from zero to well above the percolation threshold.

Trompette et al. [24] reported $G^{\prime} \sim c_{a}^{3.3}$ for PA gels with various $c_{a} / c_{\mathrm{bis}}$ ratios, which is close to our own studies furnishing $G^{\prime} \sim c_{a}^{3.5}$ (to be reported elsewhere). Weiss and Silberberg [10] studied the crosslinking of PA, determining an optimum bis concentration $c_{\text {bis }}(\approx 2.5 \%$ bis to acrylamide molar ratio) beyond which the storage modulus $G^{\prime}$ does not increase. Calvet et al. [6] obtained $c_{\text {bis }} / c_{a} \approx 2 \%$ as the optimum crosslinker concentration at constant acrylamide concentration $c_{a}\left(8 \mathrm{w} \%\right.$ equivalent to $\left.1.13 \mathrm{~mol} \mathrm{l}^{-1}\right)$, finding that the loss modulus $G^{\prime \prime}$ for the softest gels (low $c_{\text {bis }}$ ) increased similarly to $G^{\prime}$ during gelation. Note that $G^{\prime \prime}$ was often below the instrument detection limit, so the variation of $G^{\prime \prime}$ with $c_{\text {bis }}$, which reflects the size and concentration of polymer clusters and free polymer chains, could not be resolved. Moreover, high acrylamide concentrations $c_{a}$ furnished a narrow range of viscoelastic properties (loss tangents $\tan \delta \approx 10^{-3}-10^{-2}$ ).

The present study probes polymerization kinetics and shear moduli with a much lower monomer concentration $c_{a} \approx 3 \mathrm{w} \%$ (equivalent to $0.42 \mathrm{~mol}^{-1}$ ) than in the previous studies. The experiments furnish $G^{\prime}$ and $G^{\prime \prime}$ spectra with $\tan \delta$ spanning 4 orders of magnitude. These data are analyzed to establish universal scaling exponents at the gel point. Finally, complementary strain sweeps elucidate how the crosslink density and partial-chain stretching affect fracture.

\section{MATERIALS AND METHODS}

PA hydrogels were synthesized by mixing acrylamide (monomer), received as a $40 \mathrm{w} \%$ aqueous solution (Fisher Scientific, U.S.A.), bis as crosslinker, received as a $2 \mathrm{w} \%$ aqueous solution (Fisher Scientific, U.S.A.), ammonium persulfate (APS) powder (Fisher Scientific, U.S.A.) and $\mathrm{N}, \mathrm{N}, \mathrm{N}^{\prime}, \mathrm{N}^{\prime}$-tetramethylethylenediamine (TEMED, GE Healthcare Life Science, Germany) as initiator and catalyst for the free-radical polymerization.

At a constant monomer concentration $c_{a} \approx 0.42 \mathrm{~mol}^{-1}$, the bis to acrylamide molar ratio was varied in the range $0 \%-4.6 \%$ to adjust the crosslink density. Note that $10 \mu \mathrm{l}$ APS $\left(0.44 \mathrm{~mol}^{-1}\right.$ solution) and $3 \mu \mathrm{l}$ TEMED per $\mathrm{ml}$ of pregel solution were added to all the samples. Reverse-osmosis (RO) water was used to adjust the concentrations, and 
dissolved oxygen was removed by bubbling $\mathrm{N}_{2}$ gas through mixtures for $\approx 5 \mathrm{~min}$ before adding TEMED.

To clearly distinguish the samples, we adopt a nomenclature $\mathrm{A}_{x} \mathrm{~B}_{y}$, where $x$ is the nominal molar concentration of acrylamide monomer $c_{a}$, and $y$ is the nominal value of bis crosslinker $c_{\text {bis }}\left(\mathrm{mol} \mathrm{l}^{-1}\right) / c_{a}\left(\mathrm{~mol} \mathrm{l}^{-1}\right) \times 100$. All the concentrations are based on the pregel solution volume.

Rheological properties were measured under oscillatory shear with prescribed strain amplitude $\gamma$ using an ARES-G2 rheometer (TA Instruments, U.S.A.), which measures stress and strain independently using a separate motor and transducer. Gelation proceeds with the sample sandwiched between parallel rheometer plates with a Peltier device for temperature control. The temperature was set to $21^{\circ} \mathrm{C}$, and evaporation was minimized using an "evaporation blocker" provided by the manufacturer. Gelation was monitored at an angular frequency $\omega=10 \mathrm{rad} \mathrm{s}^{-1}$ and strain amplitude $\gamma=2 \%$ or $5 \%$ (both in the linear viscoelastic range). When the properties reached a steady-state $(\approx 70 \mathrm{~min})$, a frequency sweep in the range $\omega=0.1-100 \mathrm{rad} \mathrm{s}^{-1}$ was undertaken with $\gamma=2 \%$ or $5 \%$, followed by a strain sweep $\gamma=0.1 \%-100 \%$ at $\omega=10 \mathrm{rad} \mathrm{s}^{-1}$.

\section{RESULTS AND DISCUSSION}

\section{A. Crosslinking efficiency and crosslinker effect on kinetics}

Figure 1 shows the typical response of a PA hydrogel during polymerization. After an incubation period $t_{i}$, during which the moduli are below the instrument sensitivity, the storage and loss moduli increase rapidly until they reach a plateau. Beyond the incubation period, the storage modulus data can be fit to [25]

$$
G^{\prime}(t)=\frac{G_{\infty}^{\prime} t^{\alpha}}{t^{\alpha}+\theta^{\alpha}},
$$

which is adopted here as a low-dimensional fitting model, often termed a concentration-effect curve in pharmacological applications [26]. Here $t$ is the time with the gelation half

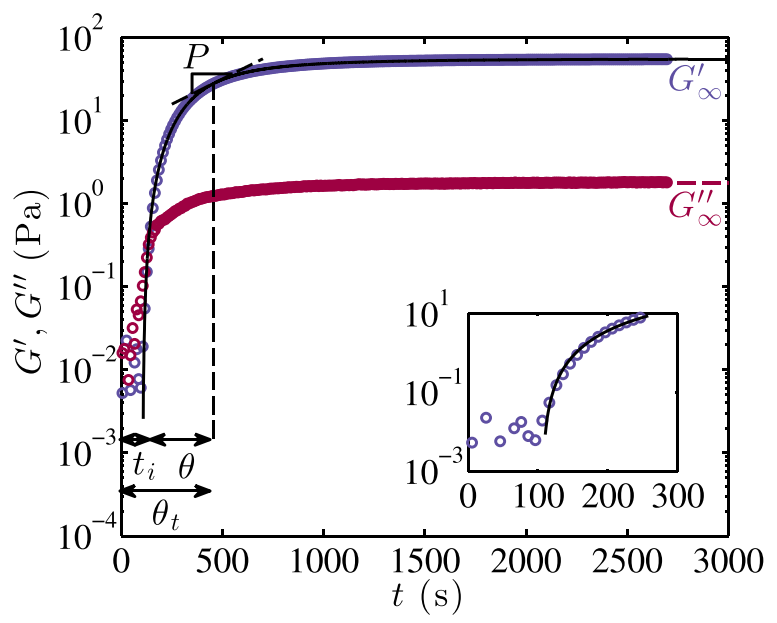

FIG. 1. Storage $\left(G^{\prime}\right)$ and loss $\left(G^{\prime \prime}\right)$ moduli time series $\left(\omega=10 \mathrm{rad} \mathrm{s}^{-1}\right)$ during polymerization of $\mathrm{A}_{0.4} \mathrm{~B}_{0.9}$ (inset is $G^{\prime}$ at short times). time $\theta\left(G^{\prime}(\theta)=G_{\infty}^{\prime} / 2\right)$, steady-state modulus $G_{\infty}^{\prime}$ and exponent $\alpha$ taken to be fitting parameters. The rate of production of elastically effective crosslinks at $\theta$ is

$$
\dot{n}_{\theta}=\frac{P}{k_{B} T},
$$

where $P=\alpha G_{\infty}^{\prime} / 4 \theta$ is the slope at $t=\theta$. The kinetic parameters according to Eqs. (2) and (3) are shown in Fig. 2 versus $c_{\text {bis. }}$. At constant $c_{a}$, increasing $c_{\text {bis }}$ reduces $\theta_{t}=t_{i}+\theta$ because of the higher reaction probability between monomers and bis. However, with $c_{\text {bis }} / c_{a} \gtrsim 2.5 \%, \theta_{t}$ is independent of $c_{\text {bis }}$, suggesting that $c_{a}$ is rate-determining. Note that $\dot{n}_{\theta}$ also increases with $c_{\text {bis }}$ when $c_{\text {bis }} / c_{a} \lesssim 2.5 \%$, but when $c_{\text {bis }} / c_{a} \gtrsim 2.5 \%$ the added crosslinks do not contribute to the elastic network because of insufficient monomer, thus resulting in a constant value of $\dot{n}_{\theta}$. Note that small values of $\dot{n}_{\theta}$ reflect very low crosslinking efficiencies $(\ll 1 \%)$, as examined below.

Measurements of Calvet et al. [6] on PA hydrogels with $c_{a} \approx 8 \mathrm{w} \%$ showed that the gelation half time depends weakly on $c_{\text {bis }}\left(\approx 1400 \%\right.$ increase in $c_{\text {bis }}$ decreases $\theta$ by less that $20 \%)$. However, our samples with $c_{a} \approx 3 \mathrm{w} \%(\tan \delta$ in the range $10^{-3}-10^{1}$ ) show that $\theta$ can be reduced by more than $50 \%$ when increasing $c_{\text {bis }}$ by $\approx 1400 \%$. This suggests that $c_{\text {bis }}$ has a much more significant impact on gelation kinetics when $c_{a}$ is small. In addition, the exponent $\alpha \approx 3.6$ for PA hydrogels with $c_{a} \approx 8 \mathrm{w} \%$ and different values of $c_{\text {bis }}$ according to Calvet et al.; nevertheless, we found that $\alpha \approx 2.05$ with $c_{a} \approx 3 \mathrm{w} \%$, suggesting that $\alpha$, which represents the rate of polymerization at constant $\theta$, is independent of $c_{\text {bis }}$, but varies with $c_{a}$, suggesting that $c_{a}$ is more influential on the kinetics than $c_{\text {bis. }}$.

\section{B. Percolation}

PA percolation is examined here by varying the gelation extent with $c_{\text {bis }}$. The storage and loss moduli spectra are shown in Fig. 3 during the sol-gel transition. Each spectrum is from a sample with different $c_{\text {bis }}$ in the frequency range $\omega=0.1-100 \mathrm{rad} \mathrm{s}^{-1}$. Without crosslinking, the response is

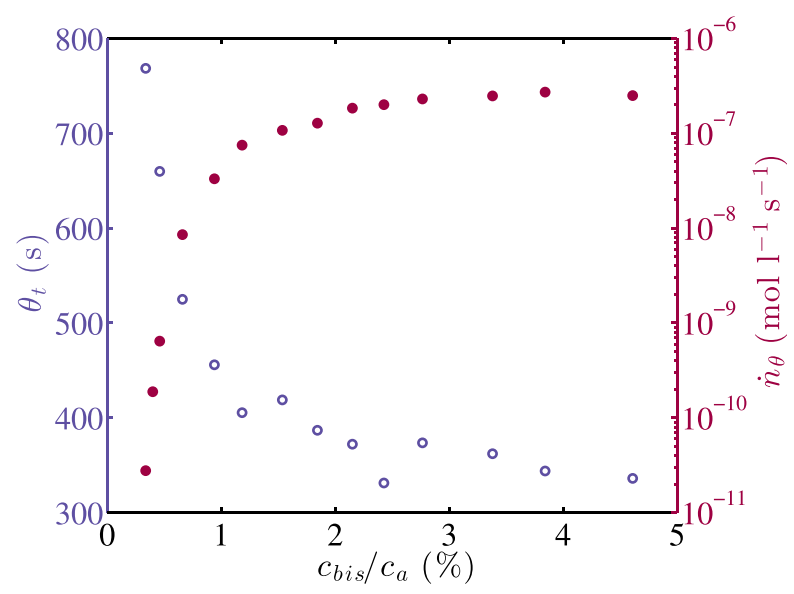

FIG. 2. Gelation half time (open symbols, left axis) and the rate of the production of crosslinks at the gelation half time (filled symbols, right axis) versus bis concentration scaled with $c_{a} \approx 0.4 \mathrm{~mol}^{-1}$. 


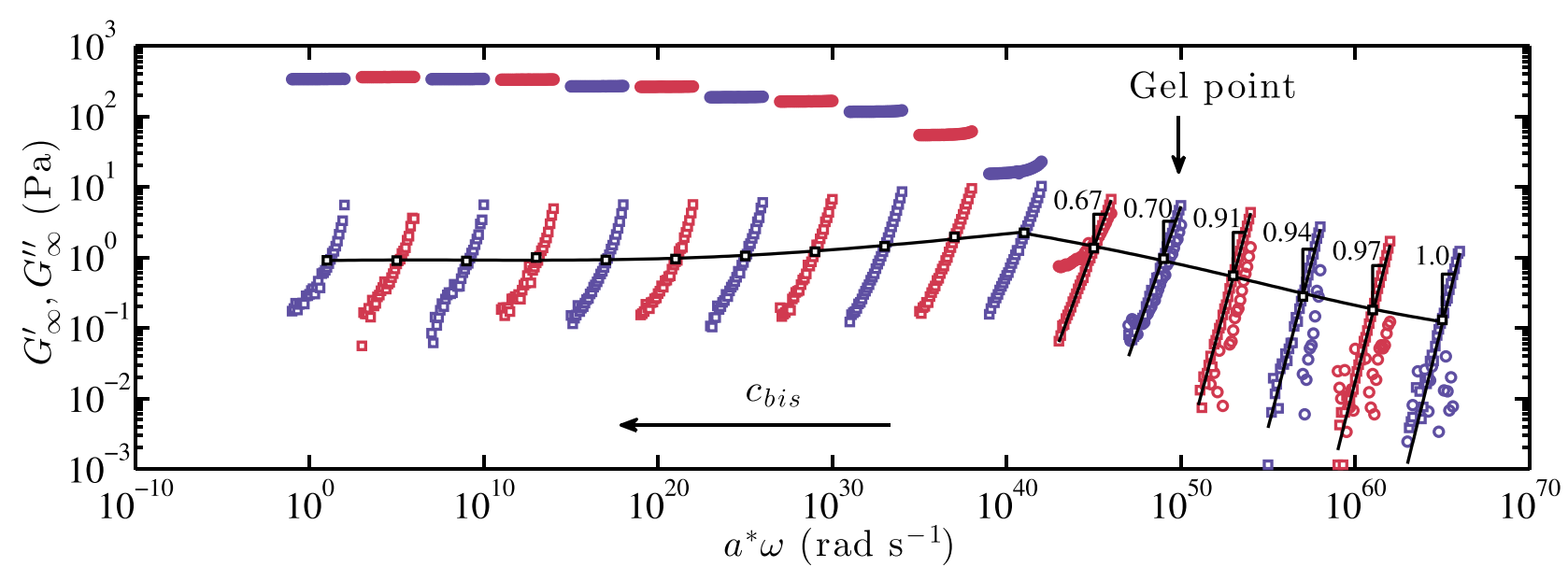

FIG. 3. Steady-state storage (circles) and loss (squares) moduli spectra (separated for clarity by an arbitrary shift factor $a^{*}$ ) for PA hydrogels with $c_{a} \approx 0.4$ mol $1^{-1}$ and the bis concentration decreasing from left to right. The polymer solution with $c_{\text {bis }}=0$ has $G_{\infty}^{\prime \prime} \sim \omega^{1}$, and the gel point occurs with $c_{\text {bis }} / c_{a} \approx 0.4 \%$ and $G_{\infty}^{\prime} \sim G_{\infty}^{\prime \prime} \sim \omega^{0.7}$. The line connects $G_{\infty}^{\prime \prime}$ values at $\omega=10 \mathrm{rad} \mathrm{s}^{-1}$.

viscous with $G^{\prime \prime} \sim \omega^{1}$, suggesting free diffusion of polymer chains. Next, increasing $c_{\text {bis }}$, which increases the cluster size, decreases the exponent due to Rouse dynamics, which increase the longest relaxation time $\tau_{l}$. At the gel point $\left(c_{\text {bis }} /\right.$ $c_{a} \approx 0.4 \%$ ), an infinite cluster is formed with $\tau_{l} \rightarrow \infty$ [9]. Finally, above the gel point, a viscoelastic solid with a lowfrequency plateau in $G^{\prime}$ is formed, and further gelation causes both $G^{\prime}$ and $G^{\prime \prime}$ to plateau. A low-frequency plateau in $G^{\prime \prime}$ is a less well known characteristic of viscoelastic solids [17], which can occur at much lower frequencies than the plateau in $G^{\prime}$. Also, close to the gel point, the longest relaxation time decreases as $\tau_{l} \sim \epsilon^{-y}$ upon further crosslinking.

It is generally understood that large clusters are formed with increasing $c_{\text {bis }}$ below the gel point, and that these clusters attach to the percolation network and decrease the viscosity above the gel point [20]. However, for our samples, $G^{\prime \prime}$ reaches a maximum at $c_{\text {bis }} / c_{a}=0.7 \%$, which is above the gel point at $c_{\mathrm{bis}} / c_{a} \approx 0.4 \%$. Mours and Winter [27] also found a maximum in $G^{\prime \prime}$ for crosslinked polybutadiene above the gel point, and in a review paper Mours and Winter [28] reported that the maximum value of $G^{\prime \prime}$ occurs far beyond the gel point, contrary to the theoretical expectations of Martin et al. [20]. Thus, a maximum in $G^{\prime \prime}$ does not identify the gel point, because $G^{\prime \prime}$ decreases only when fewer clusters are formed than are connected to the network.

The measured gel point of Larsen and Furst [9] ( $c_{\text {bis }} \approx 0.062$ wt. $\%$, equivalent to $c_{\text {bis }} / c_{a} \approx 0.96 \%$ ) for PA with $c_{a} \approx 0.4 \mathrm{~mol} 1^{-1}$ is much higher than ours $\left(c_{\text {bis }} /\right.$ $c_{a} \approx 0.4 \%$, equivalent to $c_{\text {bis }} \approx 0.026$ wt. $\%$ ), with a smaller critical relaxation exponent $\Delta(0.55 \pm 0.03$ versus our 0.70 \pm 0.02 ). From our bulk rheology, $G^{\prime}$ and $G^{\prime \prime}$ for the PA gel with $c_{a} \approx 0.4 \mathrm{~mol} \mathrm{l}^{-1}$ and $c_{\text {bis }} / c_{a} \approx 0.9 \%$ (eighth curve from the right in Fig. 3) both have low-frequency plateaus, and the sample is not close to the gel point. Note also that (i) a plateau in $G^{\prime}$ is present with even smaller values of $c_{\text {bis }}$, and (ii) $G^{\prime}$ and $G^{\prime \prime}$ both have a single power-law frequency dependence at the percolation threshold $\left(c_{\text {bis }} / c_{a} \approx 0.4 \%\right.$, equivalent to 0.026 wt. $\%$ bis) with critical exponent $n \approx 0.7$. The difference in the reported gel points may be due to difficulties in determining the gel point using a single MSD curve from microrheology, whereas we have the advantage of identifying the gel point using $G^{\prime}$ and $G^{\prime \prime}$ spectra.

Figure 4 shows the master curve constructed by shifting the dynamic moduli spectra with $c_{\text {bis }} / c_{a} \geq 0.4 \%$ (above the gel point). The spectra are shifted with factors $a \sim \tau_{l} \sim \epsilon^{-y}$ and $b \sim G^{-1} \sim \epsilon^{-z}$ [23] along the abscissa and ordinate, respectively. The resulting master curve shows the lowfrequency plateau for $G^{\prime}$ and $G^{\prime \prime}$ at low $\tau_{l} \omega$, and the frequency-independent power-law scaling of $G^{\prime}$ and $G^{\prime \prime}$ with

$$
\Delta=0.70 \pm 0.02
$$

at high $\tau_{l} \omega$. Unfortunately, we were unable to construct a master curve for the dynamic moduli spectra of solutions below the gel point $\left(c_{\text {bis }} / c_{a} \leq 0.4 \%\right)$. However, according to Adolf and Martin, the scaling exponents $z$ and $y$ are equal before and after the gel point; therefore, one can obtain the scaling exponents from data either below or above the gel point, and use $\Delta=z / y$ as a consistency check [19]. Note

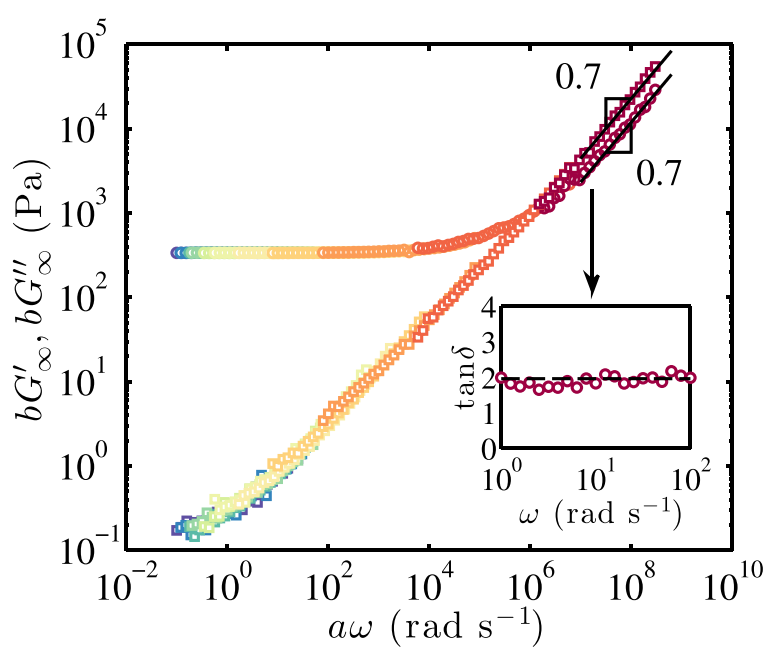

FIG. 4. Superposition of the storage (circles) and loss (squares) moduli spectra from Fig. 3 above the gel point. Frequency and moduli have been scaled with shift factors $a$ and $b$ that are available in Fig. 5. The inset shows the loss tangent versus frequency at the percolation threshold (dashed line is the theoretical value $\tan \delta=\tan (\pi \Delta / 2) \approx 1.96)$. 
also that the measured value of $\tan \delta$ at the percolation limit (shown in the inset of Fig. 4) is consistent with the theory [18]

$$
\tan \delta=\tan (\pi \Delta / 2) \approx 1.96
$$

suggesting that $\tan \delta$ depends only on $\Delta$ (not the frequency) at the gel point.

The bond probability $p \sim c_{b i s}$ when close to the gel point, since $n$ (elastically effective crosslink number density), changes linearly with $c_{\text {bis }}$ when $c_{\text {bis }} / c_{a}<2.5 \%$, as shown in Fig. $7\left(G^{\prime} \approx n k_{B} T\right)$. The shift factors $a$ and $b$ plotted in Fig. 5 versus the distance from the gel point

$$
\epsilon=\frac{\left|c_{\text {bis }}-c_{\text {bis }, p}\right|}{c_{\text {bis }, p}}
$$

have power-law scaling ( $c_{\mathrm{bis}, p}$ is the critical bis concentration at the gel point). The scaling exponents from these data,

$$
y=3.1 \pm 0.1 \text { and } z=2.1 \pm 0.1
$$

are in good agreement with the values of Larsen and Furst [9] for PA pregel solutions $(y=3.6 \pm 0.1$ and $z=2.3 \pm 0.1)$. These are also close to the values obtained by Adam and Delsanti [22] for the radical copolymerization of a different polymer $(z=2.1 \pm 0.3$ and $k=0.78 \pm 0.05$, giving $y \approx 2.9$ and $\Delta \approx 0.72$ ).

Dynamics at the gel point depend significantly on the polymer molecular weight when crosslinking polymers from high-molecular-weight precursors [27]. For example, when gels were prepared by end-linking high molecular weight polymers, the critical behavior may shrink to a small and experimentally inaccessible regime, so scaling behavior cannot be measured at the gel point [19]. In contrast, radical polymerization produces polymer chains with a broad range of molecular weights. Different materials formed by radical polymerization can have similar molecular-weight distributions and cluster structures during polymerization, which perhaps explains why they exhibit similar characteristics

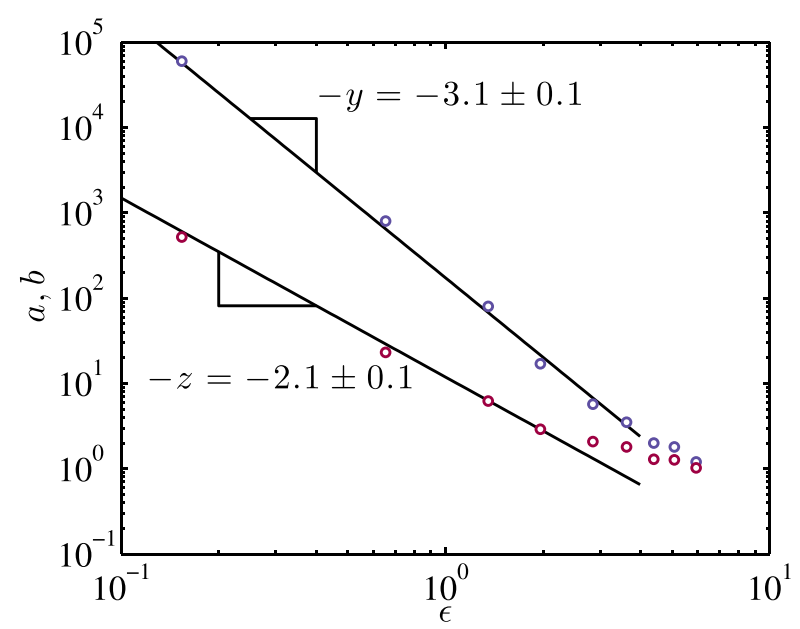

FIG. 5. Shift factors $a$ (top) and $b$ (bottom) versus distance from the gel point. Lines $\left(a \approx 173.1 \epsilon^{-3.1}\right.$ and $\left.b \approx 11.9 \epsilon^{-2.1}\right)$ are fits the power-law region with $\epsilon \lesssim 2$. during the sol-gel transition, thus highlighting the importance of the gelation method.

Finally, note that the critical relaxation exponent for PA obtained from the foregoing values of $z$ and $y$,

$$
\Delta=z / y=0.68 \pm 0.05
$$

is in good agreement the value $(\Delta=0.70 \pm 0.02)$ obtained directly from the shear-moduli spectra at the gel point. This value is also close to the Rouse value $\Delta=2 / 3$ according to scaling theory of Martin et al. [19]. Finally, $z$ and $y$ are close to the de Gennes electrical network analogy $(z=1.94$, $k=0.75, \Delta=0.72$ ), thus demonstrating reasonable agreement between our experiments and the available theories based on the percolation theory.

\section{Crosslinker effect on mechanical properties}

We compared $G^{\prime}$ for an ideal network in which each bis contributes to an elastically effective crosslink, according to Eq. (1) $\left(G_{\text {ideal }}^{\prime}\right)$ with the measured storage modulus (listed in Table I). The resulting crosslinker efficiencies $G_{0 \infty}^{\prime} / G_{0, \text { ideal }}^{\prime}$ are shown in Fig. 6. Based on these, only a small fraction of crosslinks contribute to the elasticity ( $<1 \%$ for $c_{a} \approx 0.4 \mathrm{~mol}$ $1^{-1}$ ), mainly because of the accumulation of bis in clusters. Increasing the bis concentration increases the concentration of crosslinking junctions in the dilute phase that surrounds the clusters, increasing the efficiency by reducing inhomogeneity. However, when $c_{\text {bis }} / c_{a} \gtrsim 2.5 \%$, there is insufficient monomer for gelation, so elastically ineffective structures, such as dangling chains and loops, decrease the crosslinking efficiency. Note that the efficiency also depends on the monomer concentration [10]. By increasing $c_{a}$, the polymer concentration difference between the clusters and the surrounding dilute phase decreases, producing a more homogeneous network. Therefore, increasing the monomer content enhances the crosslinking efficiency, suggesting that the

TABLE I. PA hydrogel compositions and viscoelastic properties from dynamic rheology at $\omega=10 \mathrm{rad} \mathrm{s}^{-1}$ and $\gamma=2 \%$ with $c_{a} \approx 0.42 \mathrm{~mol} \mathrm{l}^{-1}$.

\begin{tabular}{lccc}
\hline \hline Sample & $c_{\text {bis }}\left(\mathrm{mmol} \mathrm{l}^{-1}\right)$ & $G_{\infty}^{\prime}(\mathrm{Pa})$ & $G_{\infty}^{\prime \prime}(\mathrm{Pa})$ \\
\hline $\mathrm{A}_{0.4} \mathrm{~B}_{4.6}$ & 19.5 & 344 & 0.8 \\
$\mathrm{~A}_{0.4} \mathrm{~B}_{3.8}$ & 16.2 & 366 & 0.8 \\
$\mathrm{~A}_{0.4} \mathrm{~B}_{3.4}$ & 342 & 0.8 \\
$\mathrm{~A}_{0.4} \mathrm{~B}_{2.8}$ & 14.3 & 337 & 0.8 \\
$\mathrm{~A}_{0.4} \mathrm{~B}_{2.4}$ & 11.7 & 270 & 0.8 \\
$\mathrm{~A}_{0.4} \mathrm{~B}_{2.2}$ & 10.2 & 265 & 0.9 \\
$\mathrm{~A}_{0.4} \mathrm{~B}_{1.8}$ & 190 & 1.0 \\
$\mathrm{~A}_{0.4} \mathrm{~B}_{1.5}$ & 9.1 & 165 & 1.2 \\
$\mathrm{~A}_{0.4} \mathrm{~B}_{1.2}$ & 7.8 & 118 & 1.4 \\
$\mathrm{~A}_{0.4} \mathrm{~B}_{0.9}$ & 6.5 & 56 & 1.9 \\
$\mathrm{~A}_{0.4} \mathrm{~B}_{0.7}$ & 5.0 & 17 & 2.1 \\
$\mathrm{~A}_{0.4} \mathrm{~B}_{0.5}$ & 4.0 & 1.6 & 1.3 \\
$\mathrm{~A}_{0.4} \mathrm{~B}_{0.4}$ & 2.8 & 0.6 & 1.0 \\
$\mathrm{~A}_{0.4} \mathrm{~B}_{0.3}$ & 1.9 & 0.1 & 0.6 \\
$\mathrm{~A}_{0.4} \mathrm{~B}_{0.2}$ & 1.7 & - & 0.3 \\
$\mathrm{~A}_{0.4} \mathrm{~B}_{0.1}$ & 1.4 & - & 0.2 \\
$\mathrm{~A}_{0.4} \mathrm{~B}_{0}$ & 1.0 & - & 0.1 \\
\hline \hline
\end{tabular}




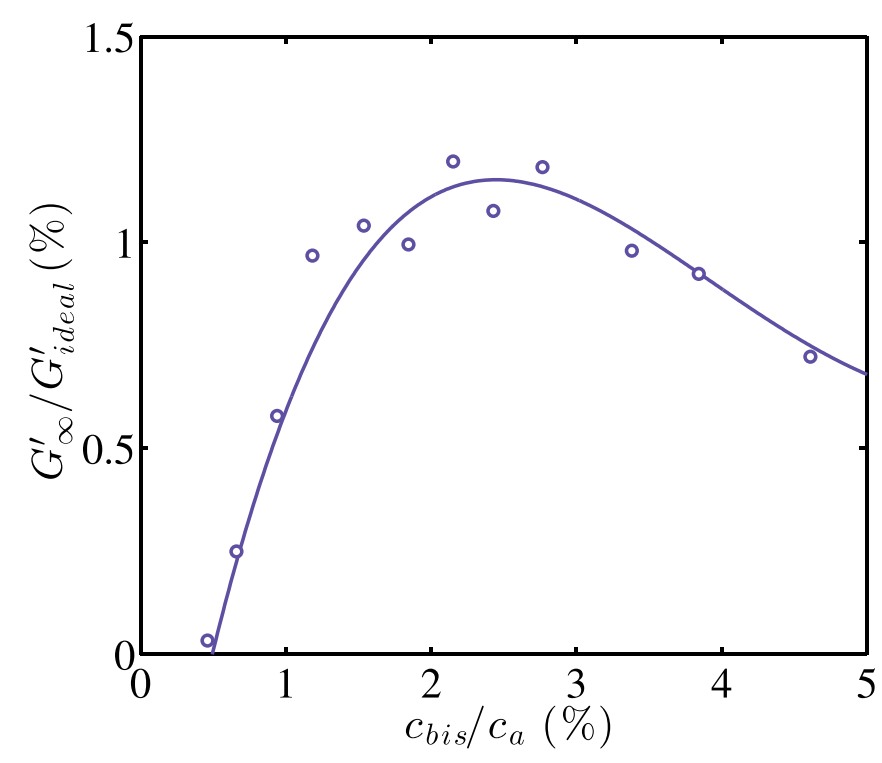

FIG. 6. Crosslinking efficiency versus bis concentration scaled with $c_{a} \approx 0.4 \mathrm{~mol}^{-1}$. The maximum is $\approx 1.2 \%$ at $c_{\text {bis }} / c_{a} \approx 2.5 \%$.

microstructures of the stiffest gels are closer to ideal networks.

The steady-state storage modulus, from the limiting lowfrequency plateaus, are shown in Fig. 7. At a fixed monomer
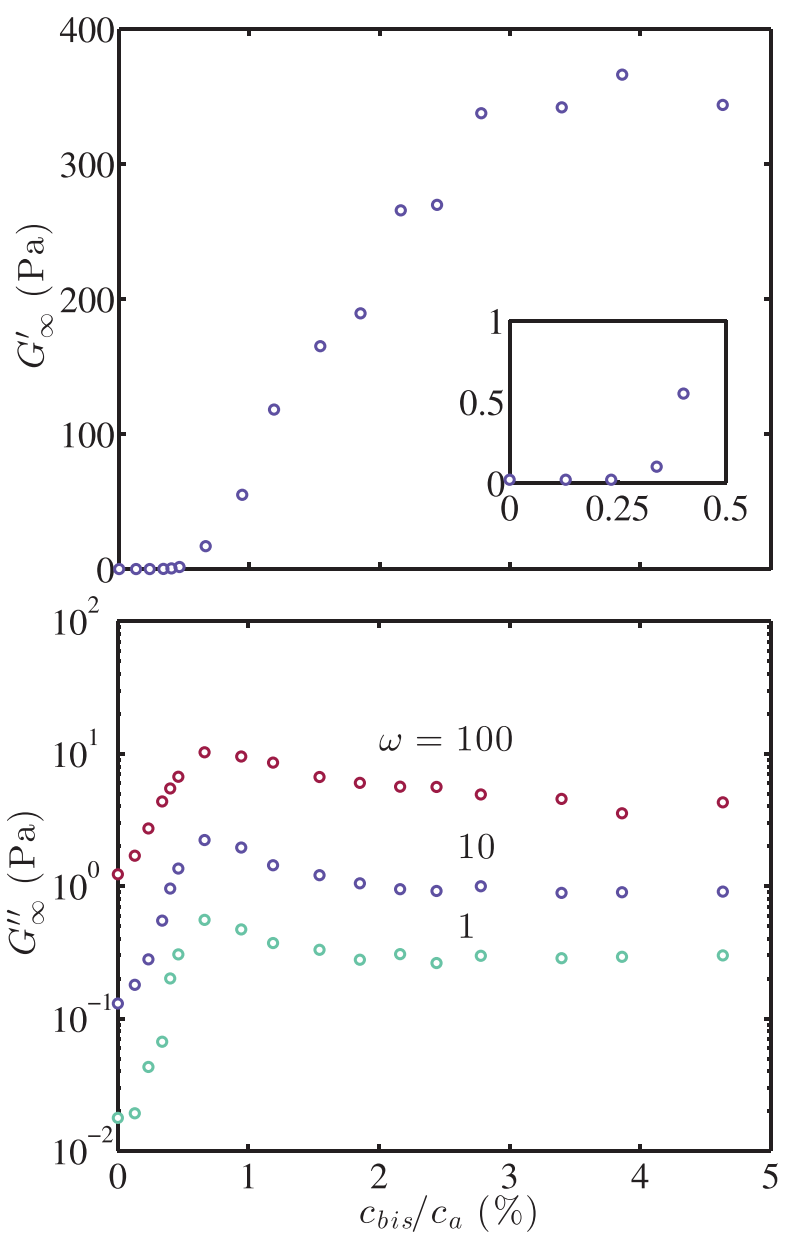

FIG. 7. Steady-state storage $\left(G_{\infty}^{\prime}\right.$, top $)$ and loss $\left(G_{\infty}^{\prime \prime}\right.$, bottom $)$ moduli of PA hydrogels versus bis concentration scaled with $c_{a} \approx 0.4 \mathrm{~mol} \mathrm{l}^{-1}$. In the bottom panel: $\omega=1$ (bottom), 10 (middle), and $100 \mathrm{rad} \mathrm{s}^{-1}$ (top). concentration, increasing $c_{\text {bis }}$ increases the crosslink density, so the growing polymer clusters form a percolating network and increase the storage modulus (see Fig. 7). However, when $c_{\text {bis }} / c_{a} \gtrsim 2.5 \%, c_{a}$ is insufficient to connect all the crosslinks, and the storage modulus does not increase further. Furthermore, dissipative properties are affected by rearrangement of polymer chains and clusters during polymerization. When $c_{\text {bis }} / c_{a} \lesssim 1 \%$, cluster growth increases the viscosity and, therefore, the loss modulus. However, when increasing $c_{\text {bis }} / c_{a}$ beyond $\sim 1 \%$, the connection of large clusters and free polymer chains to the elastic network decreases $G^{\prime \prime}$. Beyond $c_{\text {bis }} / c_{a} \approx 2.5 \%$, the attachment of large clusters to the network decreases $G^{\prime \prime}$, and the production of elastically ineffective structures enhances the energy dissipation, so a balance of these two effects produces a plateau in $G^{\prime \prime}$. Note that the loss moduli are presented at different frequencies to highlight that the maximum and plateau are frequency independent.

Increasing $c_{\text {bis }}$ beyond its optimum value causes structural changes that are often unfavorable from an engineering perspective. Brittleness of samples with different degrees of crosslinking is illustrated in Fig. 8. The maximum oscillatory strain before break $\gamma_{c}$, i.e., the strain beyond which the covalent bonds between monomers break, can be identified as the strain at which an abrupt and irreversible changes in $G^{\prime}$ and $G^{\prime \prime}$ occur. This strain is smaller for stiffer hydrogels.
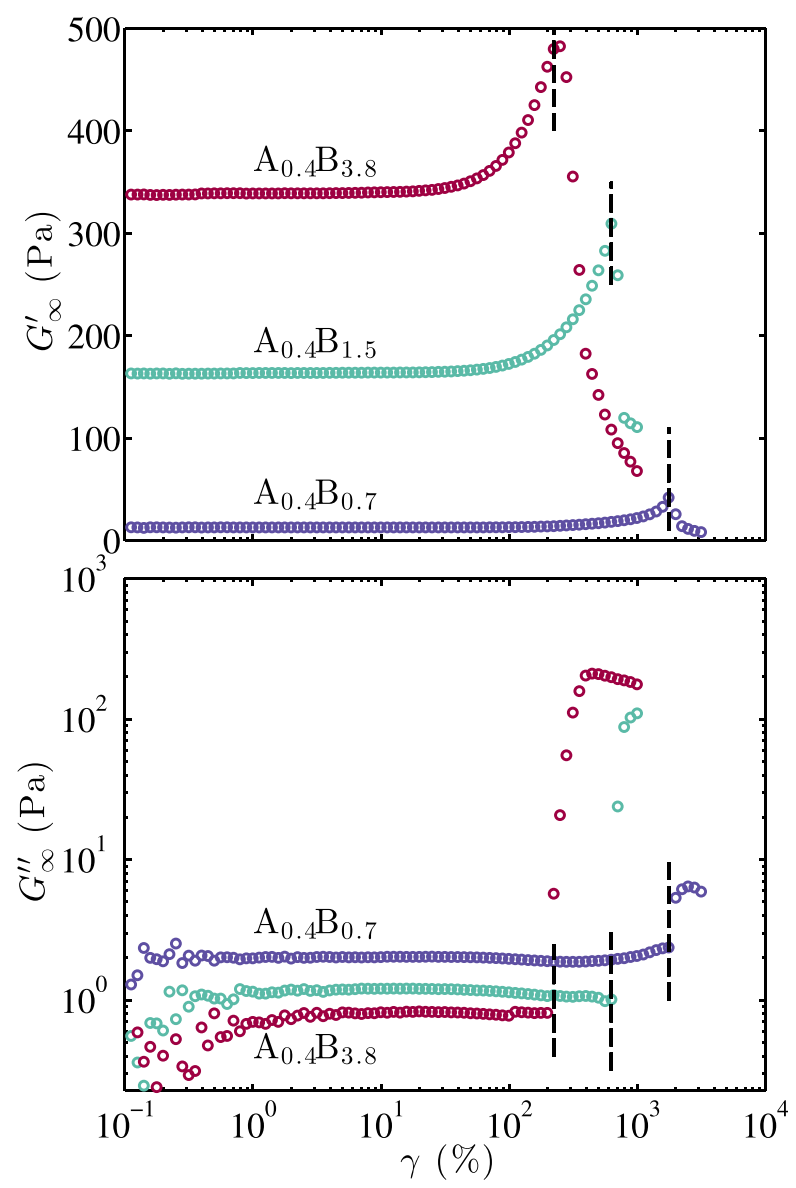

FIG. 8. Strain-sweep test for PA hydrogels $A_{0.4} B_{0.7}$ (bottom/top), $A_{0.4} B_{1.5}$ (middle), and $\mathrm{A}_{0.4} \mathrm{~B}_{3.8}$ (top/bottom) in the top/bottom panel. Dashed lines identify the breaking strain $\gamma_{c}$. 


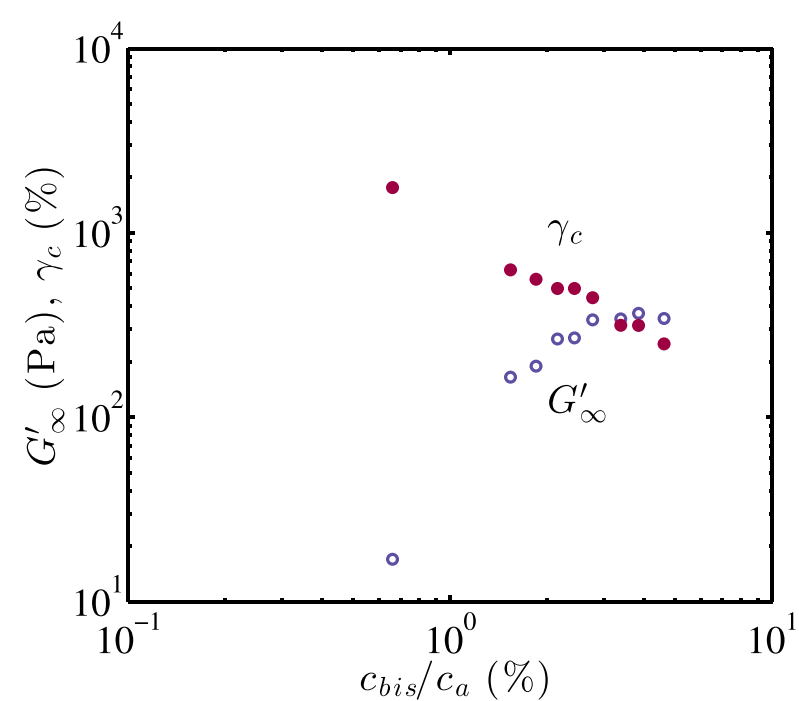

FIG. 9. Steady-state storage modulus $\left(G_{\infty}^{\prime}\right.$, open) and breaking strain $\left(\gamma_{c}\right.$, filled) and versus bis concentration scaled with $c_{a} \approx 0.4 \mathrm{~mol} \mathrm{l}^{-1}$.

The critical strain $\gamma_{c}$ is plotted versus $c_{\text {bis }}$ in Fig. 9. For soft hydrogels, polymer chains move freely between crosslinks, and can withstand larger strain without breaking. However, increasing the crosslink density produces highly stretched partial chains, so the hydrogel is more brittle with a larger storage modulus. As stated above, beyond the optimum $c_{\text {bis }}$, i.e., $c_{\text {bis }} / c_{a} \gtrsim 2.5 \%, G^{\prime}$ does not increase, as there is insufficient acrylamide to connect all the available network junctions. However, brittleness increases continuously beyond this limit, because of partial-chain stretching. Therefore, a value $c_{\text {bis }} / c_{a} \approx 2.5 \%$ is suggested to maximize PA hydrogel elasticity and minimize brittleness. Note that physical crosslinking with inorganic crosslinkers is effective in reducing hydrogel brittleness and maximizing dissipation [29].

\section{CONCLUSIONS}

Gelation kinetics and rheological properties of PA hydrogels were studied by in situ free-radical polymerization of acrylamide to ascertain the effect of chemical crosslinking on viscoelastic properties, gelation kinetics, and brittleness. In addition, time-cure superposition was used to obtain the scaling exponents at the gel point, highlighting universal behavior at the gel point.

For hydrogels with a wide range of viscoelastic properties $\left(\tan \delta\right.$ in the range $\left.10^{-3}-10^{1}\right)$, the gelation kinetics were studied by varying $c_{\text {bis. }}$. The kinetics depend on $c_{\text {bis }}$ only when $c_{\text {bis }} / c_{a} \lesssim 2.5 \%$; otherwise, $c_{a}$ is rate-determining. By comparison to results of Calvet et al. [6] with much higher $c_{a}$, the influence of $c_{\mathrm{bis}}$ on gelation kinetics is amplified when $c_{a}$ is small; for example, increasing $c_{\text {bis }}$ with $c_{a} \approx 3 \mathrm{w} \%$ enhances the gelation rate by $\approx 50 \%$.

The storage and loss moduli of PA hydrogels change differently with crosslinking. The optimum value of $c_{\text {bis }}$ to achieve the maximum crosslinking efficiency is $c_{\text {bis }} /$ $c_{a} \approx 2.5 \%$, beyond which the monomer concentration is insufficient for further crosslinking. Below this optimum value, the storage modulus increases almost linearly, while the loss modulus exhibits a maximum. This maximum, which occurs above the gel point, highlights the change in cluster size and concentration during the sol-gel transition, suggesting that cluster formation, growth, and attachment to the network occur beyond the gel point.

Above the optimum $c_{\text {bis }}$, both $G^{\prime}$ and $G^{\prime \prime}$ approach plateaus. Because of insufficient monomer, elastically ineffective structures, such as dangling chains and loops, are formed, so $G^{\prime}$ cannot be increased beyond the optimum value of $c_{\text {bis. }}$. While these nonideal structures increase $G^{\prime \prime}$, more clusters are connected to the network at higher values of $c_{\text {bis }}$, producing a plateau in $G^{\prime \prime}$. However, the hydrogel brittleness, which increases with stiffness, increases beyond the optimum value of $c_{\text {bis }}$, because of highly stretched partial chains.

The sol-gel transition was studied by gradually crosslinking a PA solution. The scaling exponents $y=3.1 \pm 0.1$ and $z=2.1 \pm 0.1$, resulting in $\Delta=z / y=0.68 \pm 0.05$, are consistent with $\Delta=0.70 \pm 0.02$ that we obtained from the slope of the moduli at the gel point. These values agree with the de Gennes electrical network analogy, while the critical relaxation exponent is close to the Rouse limit $\Delta=2 / 3$ according to the scaling theory of Martin et al. [19]. Although the universality of the scaling exponents at the gel point has been controversial, the exponents obtained by Adam and Delsanti [22] for radical copolymerization of a different polymer are in reasonable agreement with our data for PA, suggesting that dynamics at the gel point are indeed universal for different materials having similar gelation mechanisms.

\section{ACKNOWLEDGMENT}

Financial support from NSERC Discovery and NSERC Research Tools and Instruments Programs is gratefully acknowledged. V.A. was supported, in part, by a McGill Engineering Doctoral Award (MEDA). R.J.H. is thankful to Professor N. J. Wagner, University of Delaware, for pointing us toward the time-concentration superposition principle.

\section{References}

[1] Hoffman, A. S., "Hydrogels for biomedical applications," Adv. Drug Delivery Rev. 54, 3-12 (2002).

[2] Drury, J. L., and D. J. Mooney, "Hydrogels for tissue engineering: Scaffold design variables and applications," Biomaterials 24, 4337-4351 (2003).

[3] Rabillouda, T., M. Chevalletb, S. Lucheb, and C. Lelongb, "Twodimensional gel electrophoresis in proteomics: Past, present and future," J. Proteomics 73, 2064-2077 (2010).

[4] Deligkaris, K., T. Sh. Tadele, W. Olthuis, and A. Berg, "Hydrogel-based devices for biomedical applications," Sens. Actuators B 147, 765-774 (2010)

[5] Qiu, Y., and K. Park, "Environment-sensitive hydrogels for drug delivery," Adv. Drug Delivery Rev. 64, 49-60 (2012).

[6] Calvet, D., J. Y. Wong, and S. Giasson, "Rheological monitoring of polyacrylamide gelation: Importance of cross-link density and temperature," Macromolecules 37, 7762-7771 (2004). 
[7] Wang, J., and V. M. Ugaz, "Using in situ rheology to characterize the microstructure in photopolymerized polyacrylamide gels for DNA electrophoresis," Electrophoresis 27, 3349-3358 (2006).

[8] Savart, T., C. Dove, and B. J. Love, "In situ dynamic rheological study of polyacrylamide during gelation coupled with mathematical models of viscosity advancement," Macromol. Mater. Eng. 295, 146-152 (2010).

[9] Larsen, T. H., and E. M. Furst, "Microrheology of the liquid-solid transition during gelation," Phys. Rev. Lett. 100, 146001 (2008).

[10] Weiss, N., and A. Silberberg, "Inhomogeneity of polyacrylamide gel structure from permeability and viscoelasticity," Br. Polym. J. 9, 144-150 (1977).

[11] Lin, W. C., W. Fan, A. Marcellan, D. Hourdet, and C. Creton, "Large strain and fracture properties of poly(dimethylacrylamide)/silica hybrid hydrogels," Macromolecules 43, 2554-2563 (2010).

[12] Denisin, A. K., and B. L. Pruitt, "Tuning the range of polyacrylamide gel stiffness for mechanobiology applications," ACS Appl. Mater. Interfaces (published online).

[13] Baselga, J., M. A. Llorente, I. Hernandez, and I. F. Pierola, "Polyacrylamide gels. Process of network formation," Eur. Polym. J. 25, 477-480 (1989).

[14] Naghash, H. J., and O. Okay, "Formation and structure of polyacrylamide gels,” J. Appl. Polym. Sci. 60, 971-979 (1996).

[15] Baselga, J., M. A. Llorente, J. L. Nieto, I. Hernandez, and I. F. Pierola, "Polyacrylamide networks. Sequence distribution of crosslinker," Eur. Polym. J. 24, 161-165 (1988).

[16] Rubinstein, M., and H. Colby, Polymer Physics (Oxford University, New York, NY, 2003).

[17] Ferry, J. D., Viscoelastic Properties of Polymers, 3rd ed. (John Wiley \& Sons, New York, NY, 1980).
[18] Chambon, F., and H. Winter, "Linear viscoelasticity at the gel point of a crosslinking PDMS with imbalanced stoichiometry," J. Rheol. 31, 683-697 (1987).

[19] Martin, J. E., D. Adolf, and J. P. Wilcoxon, "Viscoelasticity near the sol-gel transition,” Phys. Rev. A 39, 1325-1332 (1989).

[20] Martin, J. E., D. Adolf, and J. P. Wilcoxon, "Viscoelasticity of nearcritical gels," Phys. Rev. Lett. 61, 2620-2623 (1988).

[21] de Gennes, P. G., "On a relation between percolation theory and the elasticity of gels," J. Physique Lett. 37, 1-2 (1976).

[22] Adam, M., and M. Delsanti, "Mechanical measurements in the reaction bath during the polycondensation reaction, near the gelation threshold," Macromolecules 18, 2285-2290 (1985).

[23] Adolf, D., and J. E. Martin, "Time-cure superposition during crosslinking," Macromolecules 23, 3700-3704 (1990).

[24] Trompette, J. L., E. Fabregue, and G. Cassanas, "Influence of the monomer properties on the rheological behavior of chemically crosslinked hydrogels," J. Polym. Sci. Part B 35, 2535-2541 (1997).

[25] Hill, A. V., "The combinations of haemoglobin with oxygen and with carbon monoxide,” Biochem. J. 7, 471-480 (1913).

[26] Giraldo, J., N. M. Vivas, E. Vila, and A. Badia, "Assessing the (a)symmetry of concentration-effect curves: Empirical versus mechanistic models," Pharmacol. Ther. 95, 21-45 (2002).

[27] Mours, M., and H. H. Winter, "Relaxation patterns of nearly critical gels," Macromolecules 29, 7221-7229 (1996).

[28] Winter, H. H., and M. Mours, "Rheology of polymers near liquid-solid transitions," Adv. Polym. Sci. 134, 165-234 (1997).

[29] Haraguchi, K., "Nanocomposite hydrogels," Curr. Opin. Solid State Mater. 11, 47-54 (2007). 